\title{
Effects of complexity-determined system pausing on learning from multimedia presentations
}

\section{Tzu-Chien Liu}

Department of Educational Psychology and Counseling; Institute for Research Excellence in Learning Sciences, National Taiwan Normal University, Taiwan

\section{Yi-Chun Lin}

Department of Educational Psychology and Counseling, National Taiwan Normal University, Taiwan

\author{
Slava Kalyuga \\ School of Education, University of New South Wales, Australia
}

\begin{abstract}
System pausing at pre-determined positions during multimedia presentations can enhance multimedia learning. However, the pause positions are usually set up based on the structure of the learning material (e.g., segmentation principle) rather than on the complexity of its different sections (as determined by levels of element interactivity, according to cognitive load theory). This study investigated the effectiveness of complexity-determined system pauses positioned either before or after complex (high element interactivity) sections of a slideshow multimedia presentation. The study adopted a single-factor between-subjects design and randomly assigned 128 undergraduates to four experimental conditions, namely (1) pausing before high element interactivity, (2) pausing after high element interactivity, (3) learner pausing and (4) no pausing. The research results revealed that complexity-determined system pausing approaches and learner pausing resulted in better test performance and instructional efficiency than the continuous presentation without pausing. The findings suggest that pauses allow students more time to deal with learning contents with high element interactivity, thus reducing potential cognitive overload and resulting in better performance compared with continuous presentation. However, no significant difference was found between the two types of system pausing and learner pausing in all measures.
\end{abstract}

\section{Implications for practice or policy:}

- Instructional designers could insert pauses before or after learning contents with high complexity when designing dynamic multimedia presentations.

- Instructional designers could combine system pausing and learner pausing when designing dynamic multimedia presentations.

- Instructional designers and educational system developers could develop automated systems for adding complexity-determined system pauses to learning materials based on real-time evaluation of the level of complexity.

Keywords: cognitive load theory, system pausing, learner pausing, multimedia, element interactivity, quantitative

\section{Introduction}

Cognitive overload induced by processing dynamic, rapidly changing information often occurs in learning with multimedia presentations (Khacharem et al., 2015). According to cognitive load theory (Sweller, 2020; Sweller et al., 2011), limited processing capacity (only a few elements of information at a time) and duration (only a few seconds) of working memory are the major factors influencing the effectiveness of learning materials and tasks. Dynamic multimedia presentations use various dynamic forms of visual and verbal representations, such as videos or animations with narrated verbal explanations. Such information is transient - it disappears shortly after having been displayed or spoken. The transient nature of dynamic visual and verbal representations (Berney \& Bétrancourt, 2016; Wong et al., 2020) makes it difficult for learners to process and integrate different forms of such information within limited time without overloading working memory (Biard et al., 2018; Merkt et al., 2018). 
Using system- or learner-controlled pauses is considered as an effective way of dealing with the transient nature of dynamic multimedia presentations (Castro-Alonso et al., 2021). However, there are unresolved questions about the placing of pauses in multimedia presentations and factors influencing such placing (Lee et al., 2020). The current study proposed a new type of system-controlled pauses - the complexitydetermined pauses that are placed according to the complexity (or levels of element interactivity, according to the terminology of cognitive load theory) of different sections in learning materials. Two variations of such complexity-determined pauses were investigated - the pauses positioned either before or after complex (high element interactivity) sections of multimedia presentations. The effects of these two types of system pausing approaches on learning performance, cognitive load and instructional efficiency were compared with those of learner pausing and an equivalent continuous multimedia presentation without pausing. The following sections review the theoretical bases for the proposed two types of complexitydetermined system pausing approaches and present the hypotheses for the experiment.

\section{Studies on pausing multimedia presentations}

There are two major types of pausing in multimedia presentations - learner pausing and system pausing (Wouters et al., 2007). Learner pausing adopts a learner-controlled approach to pausing multimedia presentations, and it is regarded as a means of personalising learning (e.g., Hasler et al., 2007; Höffler \& Schwartz, 2011; Schwan \& Riempp, 2004). Some instructional principles were developed based on this approach, such as interactivity principle (Bétrancourt, 2005) and self-pacing principle (van Merriënboer \& Kester, 2014). Most studies on this approach showed that giving learners control over the pace of multimedia learning could reduce the negative effects caused by the transient nature of multimedia material compared with continuous presentations (e.g., Hasler et al., 2007; Schwan \& Riempp, 2004).

However, since a learner is the key actor in the learner pausing approach, large individual differences in the use of pausing during learning might directly influence the effectiveness of this approach (Tabbers \& de Koeijer, 2010). In addition, a number of studies found very limited (if any) use of a pause function by some learners (e.g., Biard et al., 2018; Hasler et al. 2007). A possible reason for this finding is that pausing at appropriate positions is highly dependent on learners' ability to monitor their own learning, but novice learners normally lack such ability (Biard et al., 2018).

System pausing, the other approach to pause multimedia presentations, occurs when the presentations stop automatically at pre-determined positions, and it is the system rather than learners that controls the position of pauses (Merkt et al., 2018). Most of the studies of this approach have inserted pause positions based on the structure of learning contents (i.e., the system pausing by segmentation) (e.g., Biard et al., 2018; Hasler et al., 2007; Mayer \& Chandler, 2001; Spanjers et al., 2010). For example, in the study of Biard et al. (2018), a video was used to explain the procedure of making hand orthoses by steps with system pausing at the end of each step, and participants could move to the next step with the "Play" button. Another example is the study of Mayer and Chandler (2001), in which the phenomenon of lighting was explained using an animation presenting 16 segments. Pauses were inserted at the end of each segment, and participants could determine when to go to the next segment by clicking on the "Play" button. The pauses inserted between the pre-determined sections are supposed to give learners more time to review and consolidate the information of each major section to avoid the risk of cognitive overload when processing and integrating new incoming information (Spanjers et al., 2010). The system pausing by segmentation is deemed useful for learning contents with explicit structures, such as well-defined procedures (e.g., Biard et al., 2018) or concepts that could be segmented into several parts (e.g., Mayer \& Chandler, 2001).

In summary, learner pausing is regarded as an effective way for avoiding cognitive overload caused by continuous multimedia presentations (e.g., Hasler et al., 2007; Schwan \& Riempp, 2004). However, the notable individual differences in learners' use of pauses during learning (Tabbers \& de Koeijer, 2010) and high demands of efficient monitoring of the appropriate uses of pausing (Biard et al., 2018) made it unrealistic to guarantee the effectiveness of the learner pausing approach. On the other hand, system pausing might be a good approach for ensuring that all learners are given a break at the appropriate position by the automatic pause. However, most of the previous studies focused only on learning materials with clear structure, where positions of system pauses were determined by structural segmentation (e.g., Hasler et al., 2007; Mayer \& Chandler, 2001; Spanjers et al., 2010), which may reduce the potential value of the system pausing approach for multimedia materials without clear, well-outlined structure. 


\section{Rationales for complexity-determined system pausing}

In cognitive load theory, element interactivity is considered as the main source of cognitive load (Sweller, 2010, 2020). High levels of element interactivity could be generated by learning materials or tasks that require simultaneous processing of a large number of information elements that cannot be understood independently (Chen et al., 2017; Chen et al., 2018; Sweller, 2010, 2020). High levels of element interactivity could overload learner working memory and impede learning, especially for learners with low levels of prior knowledge (Sweller, 2010, 2020).

Cognitive overload caused by high element interactivity often occurs in multimedia learning environments that require learners to process transient pictorial and verbal representation simultaneously and to integrate them for understanding (Khacharem et al., 2015; Sweller, 2010, 2020). In such environments, learners need to maintain parts of the fleeting narrated information in working memory while searching and matching the corresponding information from the pictorial representation (frequently, also dynamic) and then integrate these representations to make sense of the instructional message (Ayres \& Paas, 2007; Kalyuga, 2009; Leahy \& Sweller, 2011). Tying the positions of system pauses to high element interactivity parts of dynamic multimedia presentations could potentially alleviate cognitive overload associated with such maintenance, search-and-match and mental integration activities.

The rationale for system pauses positioned before complex (high element interactivity) sections could be related to the isolated-interacting elements effect in cognitive load theory (Chen et al., 2017; Lin et al., 2015; Pollock et al., 2002; Sweller, 2010). Isolated-interacting elements effect occurs when presenting individual elements (without connections to other elements) in the first phase of instruction followed by presenting all the interacting elements together in the second phase, which results in more efficient learning than presenting fully interacting elements in both phases of instruction (Chen et al., 2017; Lin et al., 2015; Pollock et al., 2002; Sweller, 2010). This principle has been recently applied to avoid cognitive overload from high levels of element interactivity in e-learning environments (e.g., a simulation-assisted learning environment in Lin et al., 2015). However, it has not been adopted to the design of system pauses for optimising multimedia learning.

With the system pausing before the start of high element interactivity narration, learners could first familiarise themselves with individual pictorial elements during the pauses, and then handle transient information and integrate the interactive pictorial and verbal elements with less effort during the following narration. Based on the isolated-interacting elements effect (Chen et al., 2017; Lin et al., 2015; Pollock et al., 2002; Sweller, 2010), learners should be able to search the corresponding pictorial representation more easily during the narration, thus making more cognitive resources available for integrating activities.

The rationale for system pauses positioned after complex (high element interactivity) sections provides learners with sufficient time to process, maintain and consolidate the complex and transient information they have just studied, which is also a major cause behind the benefits of the segmentation effect (Spanjers et al., 2010; Spanjers et al., 2012). According to the time-based resource sharing model of working memory (Barrouillet \& Camos, 2007), learners could focus on either processing or maintaining tasks at a time as the two tasks share the limited attentional resource. Therefore, rapidly switching away from processing the information that they have just received will not only impede the maintenance and consolidation of the information that they have previously learnt but also negatively affect their following learning.

If pauses are inserted immediately after the narration with high element interactivity, learners would have more time to review the pictorial representation and complete the required search-and-match and integration processes and would need less mental effort for integrating the pictorial information still displayed on the screen and verbal information maintained in learner working memory. Thus, the system pause after high element interactivity should contribute to the maintenance and consolidation of the information that has just been received.

\section{Hypotheses}

The current study compared the two suggested types of complexity-determined system pausing (system pausing before or after high element interactivity), learner pausing and continuous presentation without pausing. Immediate and delayed post-tests performance, cognitive load during learning and instructional 
efficiency (an aggregated indicator of the relationship between the test performance and cognitive load) were measured. The following three hypotheses were proposed based on the literature review and theoretical rationales grounded in cognitive load theory:

- H1. Participants in both complexity-determined system pausing conditions (before and after high element interactivity) will demonstrate better performance in all measures than the participants in the continuous presentation without pausing condition.

- H2. Participants in the learner pausing condition will demonstrate better performance in all measures than the participants in the continuous presentation without pausing condition.

- H3. Participants in both systems complexity-determined pausing conditions will show better performance in all measures than the participants in the learner pausing condition.

\section{Research method}

\section{Experimental design and participants}

A single-factor between-subjects design was employed to test the hypotheses. The independent variable was the type of pausing (four experimental conditions). The dependent variables were the immediate and delayed post-tests performance, cognitive load in the learning phase and instructional efficiency indicator. The experiment was conducted from July to September in 2019. A total of 128 undergraduates from a national university in northern Taiwan were randomly assigned to four experimental groups: system pausing before high element interactivity group ( 9 males and 23 females), system pausing after high element interactivity group (11 males and 21 female), learner pausing group ( 8 males and 24 females) and continuous presentation without pausing group ( 7 males and 25 females). The mean age of the participants was 20.11. None of them had learnt about the topic before the experiment. No significant difference was found between the four groups in the scores for the prior knowledge test before the intervention, $F(3,124)$ $=0.82, p=.487$ based on the results of one-way ANOVA. All participants were required to read and sign an informed consent form detailing the purpose of this study and their rights as participants.

\section{Learning materials}

The learning material for the study was revised from an earlier version (Liu et al., 2021) on the topic of mindfulness. This multimedia slideshow presentation is composed of 27 slides explaining concepts of mindfulness and the brain knowledge related to mindfulness using pictorial representations and accompanying narrations (2044 words; $7 \mathrm{~min} 25 \mathrm{~s}$ in duration for the continuous presentation). Eight of the 27 slides were identified as "high element interactivity" sections according to the definition of element interactivity (Chen et al., 2017; Sweller 2010, 2020). For each of these eight sections, learners had to process several interconnected elements of pictorial and verbal information simultaneously and integrate them for understanding. The classification of these eight slides as high element interactivity sections was confirmed by three experts who were familiar with element interactivity concept and instructional design. Figure 1 provides examples of a high element interactivity section (slide) and a low element interactivity section (slide) used in the study. Figure 1(a) explains the signal transmission processes between the amygdala and hypothalamus in the fight-or-flight response using pictorial representation and narration. Figure 1(b) elaborates on the meaning of mindfulness. 


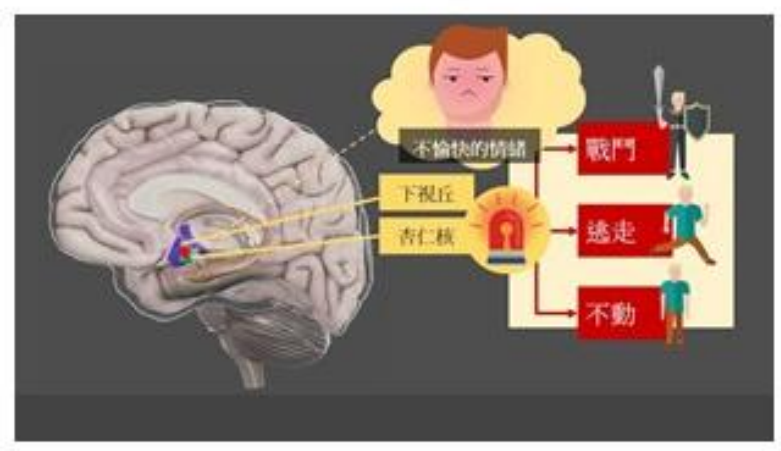

(a) high level of element interactivity
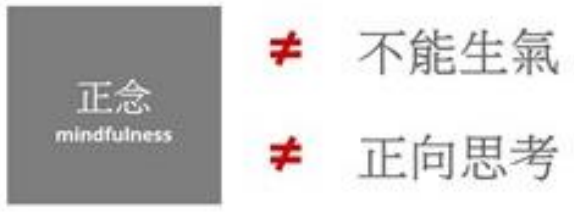

(5))

The amygdala is like a sentry

security for human beings. If it

detects that a person is in

danger, it will produce

unpleasant emotions. Then it

will activate the emergency

button to send one of the three

commands of fight, flight or

freeze to the hypothalamus

which delivers hormones to

related body parts for

promoting the production of

hormone to make preparations.

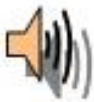

Undoubtedly, mindfulness is

not equal to can't be angry

or positive thinking

(b) low level of element interactivity

Figure 1. Examples of sections with high (a) and low (b) levels of element interactivity (the narration texts were translated from Mandarin Chinese).

The content of the learning materials for the four groups was identical, and the only difference between the groups was whether there were pauses in the multimedia presentation and the pausing type. In the system pausing before or after high interactivity groups, the presentation was automatically paused before the start of narration or after the end of narration for the eight sections (slides) with high element interactivity. Participants were reminded to continue learning after studying the contents of the section by a note next to the "Play" button. It should be noted that the participants in the complexity-determined system pausing groups were not allowed to pause the presentation at points of their choice - they could only press the "Play" button to continue the presentation after the pauses. In the learner pausing group, participants were allowed to pause and play the presentation whenever they wanted. In the continuous presentation without pausing group, participants were not allowed to pause the presentation at all.

\section{Measures}

The pause behaviours were traced, and the corresponding data were collected with logs and screen recordings. The log file included all events that occurred while participants were interacting with the presentation (e.g., pauses taken) and the timestamps for each event (e.g., pauses taken and play taken). The number of pauses and the duration of each pause were calculated from the log files for the learner pausing group and both system pausing groups. Each pause shown on the log file was recorded as one instance of pause, and the timestamps of each pause and the following play were used to calculate the duration of each pause (timestamp of the following play minus timestamp of pause). The durations of all pauses were added to obtain a total pause duration of each participant. The screen recording files captured the exact look of slides when each pause was taken in the sections with high or low element interactivity levels by participants in the learner pausing group. 
The prior knowledge test, immediate and delayed post-tests were revised versions of previously used tests (Liu et al., 2021). The revised versions of all tests were reviewed and revised by three experts who were familiar with the topic of mindfulness. The prior knowledge test was used to measure participants' prior knowledge of basic concepts of mindfulness. It included 10 multiple-choice questions (e.g., "Which one of the following statements about mindfulness meditation practice is false?"), with one point allocated for each correctly answered item (maximum score of 10 for the test). The immediate post-test was used to evaluate participants' understanding of the concepts of mindfulness and knowledge of the brain processes related to mindfulness. There were 38 items in the immediate post-test, consisting of nine matching items and 29 multiple-choice questions (e.g., When a human encounters a life-threatening emergency, the brain will activate a set of associated mechanisms to respond to this situation. Which one of the following statements about this mechanism is true?). One point was given for each correctly answered item (with a maximum possible score of 38 for the test). The Kuder-Richardson formula 20 (KR-20) which was frequently used for testing internal consistency of the items scored right versus wrong (Fraenkel et al., 2011) revealed a moderate internal consistency reliability of 0.66 . The delayed post-test was used to assess the level of retention of the participants' understanding of the learning material. The content of the delayed post-test was identical to the immediate post-test except for the sequence of the items. The internal consistency reliability of the delayed post-test (KR-20) was 0.75 .

A mental effort rating scale was applied to measure the level of cognitive load during learning. The cognitive load rating scale was a revised version of the scale developed by Paas and Van Merriënboer (1994). It was composed of three 9-point Likert items to measure participants' mental effort invested in remembering the learning content understanding the learning content, and processing the multiple sources of information during learning. The scale ranged from 1 point (very very low) to 9 points (very very high). The internal consistency reliability of mental effort rating scale (Cronbach's alpha) was 0.76.

The instructional efficiency (E) was calculated by a combination of standardised scores of mental effort participants invested in the learning phase $(\mathrm{C})$ and test performance $(\mathrm{P})$ adopting Paas and Van Merriënboer's (1993) formula:

$$
\mathrm{E}=(\mathrm{P}-\mathrm{C}) / \sqrt{2}
$$

In this study, the instructional efficiency was calculated separately for the immediate and delayed post-tests respectively. Higher levels of instructional efficiency indicate that participants learn relatively better with lower mental effort invested in the learning phase. On the contrast, lower levels of instructional efficiency indicate that participants learn relatively worse with higher mental effort invested in the learning phase.

\section{Procedure}

The experimental procedure consisted of three phases (Figure 2). In the Preparation phase, participants were informed of the purposes of this study and their rights to quit the experiments anytime at their will. After giving their consent to participate, the participants provided their personal information and took the prior knowledge test. Finally, in order to become familiar with the learning task formats in the corresponding conditions of the intervention phase, the participants went through a sample presentation with equivalent control tools (if any) on a different learning topic.

In the Intervention phase, participants studied the corresponding multimedia presentations according to their randomly assigned conditions. Similar to the study of Hasler et al. (2007) which also compared the effects of system pausing, learner pausing and no pausing conditions, the learning time in the current experiment was set the same for the four groups to ensure that the potential differences in results would not be influenced by different study period lengths. Participants in all groups were given 12 min to complete the learning task. This time limit was determined by a pilot study with five students from the same population who were not participants in the main experiment (all participants in the pilot study were able to complete the learning task within $12 \mathrm{~min}$ ). The presentation was set to play repeatedly till the end of the time limit if the first round finished earlier, and participants were not allowed to go to the next phase until the end of the time limit. The mental effort rating scale was administered after the presentation ended.

In the Test phase, the immediate post-test was conducted immediately following the Intervention phase. The participants in the learner pausing group who had not controlled the video were asked a question about 
the reasons why they did not use the "Pause" button. The delayed post-test was administered 1 week after the experiment.

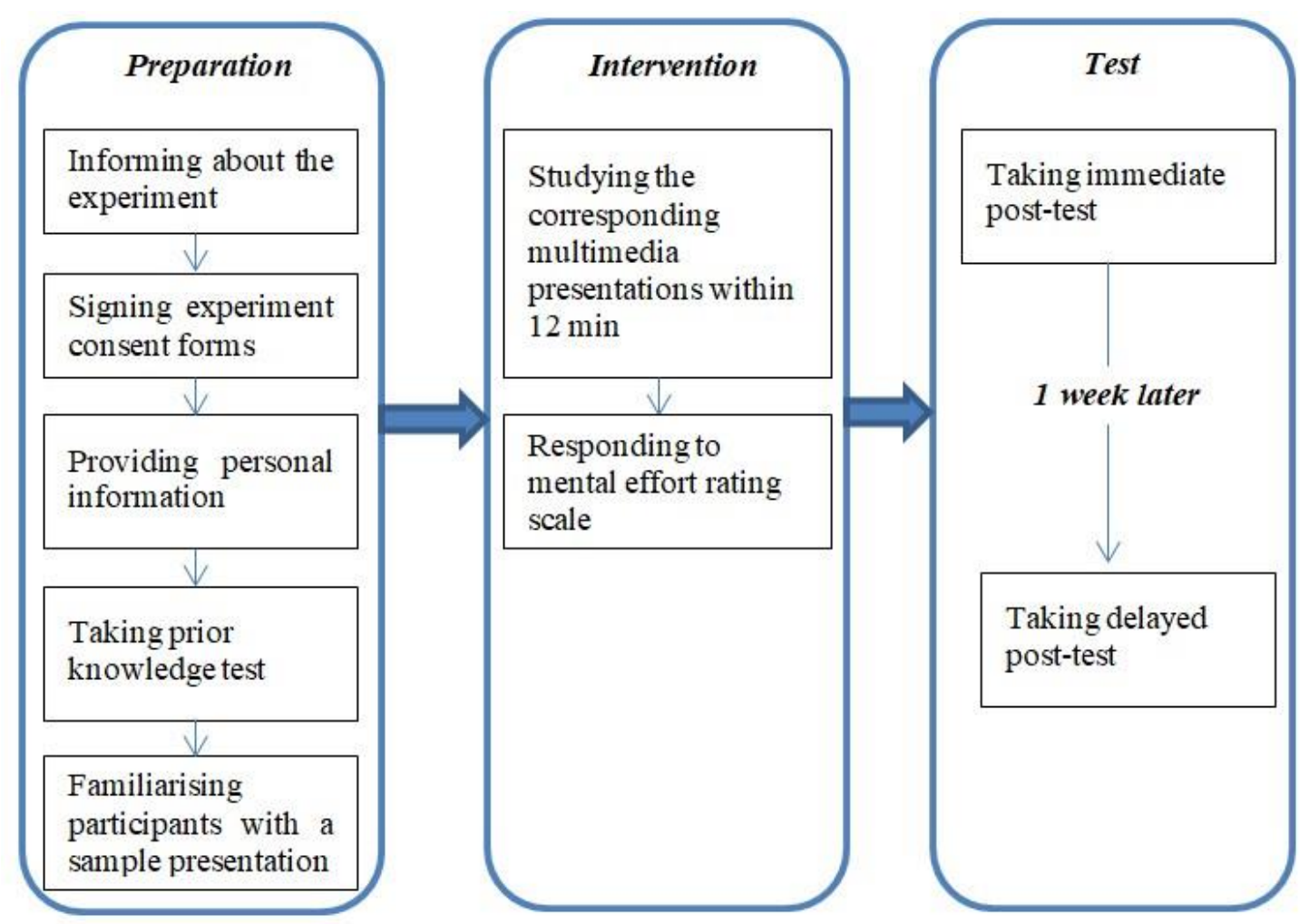

Figure 2. Experimental procedure chart

\section{Data analysis}

Since specific directional hypotheses were proposed for the differences between the experimental conditions, a set of planned contrasts was conducted to address each hypothesis (Seltman, 2018), and Cohen's $d$ was calculated to estimate the corresponding effect size. The main advantage of planned contrasts is to provide a direct and efficient way to test hypotheses of studies (Furr \& Rosenthal, 2003). In the current study, specific patterns of results for different pairs of groups were expected according to the previous studies within a framework of cognitive load theory. To address each hypothesis, the contrast weights assigned to the two groups in each contrast retained the expected pattern and summed up to zero (Furr \& Rosenthal, 2003; Rosenthal \& Rosnow, 1985). For example, to address Hypothesis 1, a series of planned contrasts was conducted with different measures to compare each type of complexity-determined system pausing with the continuous presentation condition. Accordingly, for the immediate and delayed post-test scores and instructional efficiency, the contrast weight assigned to the particular type of the complexitydetermined system pausing condition (e.g., system pausing before or after high element interactivity) was 1 , the contrast weight assigned to the continuous presentation condition was -1 , while the contrast weights assigned to each of the remaining two conditions were 0 .

\section{Results}

\section{Number of pauses and pause duration}

The number and total pause duration for the two complexity-determined types of system pausing and learner pausing groups are provided in Table 1. Since the presentation was automatically replayed until the end of time limit (12 min), for the two system pausing groups, the number of pauses inserted in the first run of the presentation was fixed (eight) and the additional number of pauses occurred in the following run(s) of the presentation depended on how much time was left. For the learner pausing group, only 10 out 
of 32 participants pressed the "Pause" button within 12 mins. The total number of the pauses initiated by these 10 participants was 33 and the total pause duration was $433 \mathrm{~s}$.

Table 1

Means and standard deviations for number of pauses and pause duration (32 participants in each group)

\begin{tabular}{|c|c|c|c|c|c|c|}
\hline \multirow[t]{3}{*}{ Dependent variable } & \multicolumn{4}{|c|}{ System pausing } & \multicolumn{2}{|c|}{ Learner pausing } \\
\hline & \multicolumn{2}{|c|}{$\begin{array}{l}\text { Pausing before high } \\
\text { element interactivity }\end{array}$} & \multicolumn{2}{|c|}{$\begin{array}{l}\text { Pausing after high } \\
\text { element interactivity }\end{array}$} & & \\
\hline & $M$ & $S D$ & $M$ & $S D$ & $M$ & $S D$ \\
\hline Number of pauses & 11.28 & 0.96 & 11.50 & 0.95 & 1.03 & 2.51 \\
\hline Total pause duration (s) & 112.78 & 31.49 & 84.50 & 33.50 & 13.53 & 30.35 \\
\hline
\end{tabular}

For the learner pausing group, further analyses of the pause positions showed that $23(69.7 \%)$ pauses occurred at slides with high element interactivity and the corresponding total pause duration was $311 \mathrm{~s}$ $(71.8 \%) ; 10(30.3 \%)$ pauses occurred at slides with low element interactivity and the total pause duration was $122 \mathrm{~s}(28.2 \%)$. Among the 10 participants who pressed the "Pause" button, six paused only once, two paused twice, three paused seven times and one paused 11 times. For the participant who paused 11 times, seven pauses (60 s in total) occurred at the slides with low element interactivity, and his learning performance was lower than the average performance in the learner pausing group.

\section{Learning performance, cognitive load and instructional efficiency}

Table 2 shows the means and standard deviations for immediate and delayed post-tests, ratings of cognitive load in the learning phase, instructional efficiency (immediate post-test) and instructional efficiency (delayed post-test).

Table 2

Means and standard deviations for immediate and delayed post-tests, ratings of cognitive load and instructional efficiency (32 participants in each group)

\begin{tabular}{|c|c|c|c|c|c|c|c|c|}
\hline \multirow[t]{3}{*}{$\begin{array}{l}\text { Dependent } \\
\text { variable }\end{array}$} & \multicolumn{4}{|c|}{$\begin{array}{c}\text { Complexity-determined system } \\
\text { pausing }\end{array}$} & \multirow{2}{*}{\multicolumn{2}{|c|}{ Learner pausing }} & \multirow{2}{*}{\multicolumn{2}{|c|}{$\begin{array}{c}\text { Continuing } \\
\text { presentation } \\
\text { without pausing }\end{array}$}} \\
\hline & \multicolumn{2}{|c|}{$\begin{array}{l}\text { Pausing before } \\
\text { high element } \\
\text { interactivity } \\
\end{array}$} & \multicolumn{2}{|c|}{$\begin{array}{c}\text { Pausing after high } \\
\text { element } \\
\text { interactivity } \\
\end{array}$} & & & & \\
\hline & $M$ & $S D$ & $M$ & $S D$ & $M$ & $S D$ & $M$ & $S D$ \\
\hline $\begin{array}{l}\text { Immediate post-test } \\
(0-38)\end{array}$ & 30.63 & 3.28 & 31.38 & 3.38 & 31.25 & 3.24 & 29.78 & 3.57 \\
\hline $\begin{array}{l}\text { Delayed post-test } \\
(0-38)\end{array}$ & 29.25 & 4.13 & 28.81 & 4.83 & 29.66 & 3.73 & 27.47 & 4.37 \\
\hline $\begin{array}{l}\text { Cognitive load in } \\
\text { the learning phase } \\
(1-9)\end{array}$ & 4.11 & 1.33 & 4.38 & 1.55 & 3.88 & 1.57 & 4.68 & 1.45 \\
\hline $\begin{array}{l}\text { Instructional } \\
\text { efficiency } \\
\text { (immediate post- } \\
\text { test) }\end{array}$ & 0.04 & 0.93 & 0.07 & 1.04 & 0.29 & 1.07 & -0.40 & 1.06 \\
\hline $\begin{array}{l}\text { Instructional } \\
\text { efficiency (delayed } \\
\text { post-test) }\end{array}$ & 0.14 & 0.94 & -0.05 & 1.17 & 0.32 & 1.01 & -0.42 & 1.24 \\
\hline
\end{tabular}

Complexity-determined system pausing vs continuous presentation without pausing

For the comparison of the system pausing before high element interactivity and continuous presentation without pausing groups, the results indicated that the system pausing group performed significantly better than the continuous presentation group in terms of the delayed post-test score, $t(124)=1.66, p=.049$, Cohen's $d=0.42$, instructional efficiency (immediate post-test), $t(124)=1.72, p=.044$, Cohen's $d=0.44$, and instructional efficiency (delayed post-test), $t(124)=2.04, p=.022$, Cohen's $d=0.51$. No significant 
differences between the two groups were found in the immediate post-test score, $t(124)=1.00, p=.159$ and cognitive load during learning, $t(124)=-1.52, p=.065$.

For the comparison of the system pausing after high element interactivity and continuous presentation without pausing groups, the results showed that the system pausing group performed significantly better than the continuous presentation group in terms of the immediate post-test score, $t(124)=1.89, p=.030$, Cohen's $d=0.46$ and instructional efficiency (immediate post-test), $t(124)=1.85, p=.033$, Cohen's $d=0.45$. No significant differences were found in the delayed post-test scores, $t(124)=1.26, p=.106$, cognitive load during learning, $t(124)=-0.82, p=.207$ and instructional efficiency (delayed post-test), $t(124)=1.33, p=$ .093 .

Learner pausing vs continuous presentation without pausing

The results of the planned contrasts for testing the differences between the learner pausing and continuous presentation without pausing groups indicated that the learner pausing group performed significantly better than the continuous presentation group in terms of immediate post-test score, $t(124)=1.74, p=.042$, Cohen's $d=0.43$, delayed post-test score, $t(124)=2.04, p=.022$, Cohen's $d=0.54$, instructional efficiency (immediate post-test), $t(124)=2.67, p=.004$, Cohen's $d=0.65$, and instructional efficiency (delayed posttest), $t(124)=2.70, p=.004$, Cohen's $d=0.65$. In addition, the learner pausing group perceived significantly lower cognitive load during learning than the continuous presentation group, $t(124)=-2.17, p=.016$, Cohen's $d=0.53$.

\section{Complexity-determined system pausing vs learner pausing}

For the comparison of the system pausing before high element interactivity and learner pausing groups, the results of planned contrasts showed no significant differences in the immediate post-test scores, $t(124)=$ $0.74, p=.230$, delayed post-test scores, $t(124)=-0.38, p=.353$, cognitive load in learning phase, $t(124)=$ $0.65, p=.259$, instructional efficiency (immediate post-test), $t(124)=-0.95, p=.172$ and instructional efficiency (delayed post-test), $t(124)=-0.66, p=.256$.

For the comparison of the system pausing after high element interactivity and learner pausing groups, the results of planned contrasts also showed that there were no significant differences between the two groups in immediate post-test scores, $t(124)=0.15, p=.441$, delayed post-test scores, $t(124)=-0.79, p=.216$, cognitive load in learning phase, $t(124)=1.35, p=.089$, instructional efficiency (immediate post-test), $t(124)=-0.82, p=.206$ and instructional efficiency (delayed post-test), $t(124)=-1.37, p=.086$.

\section{Discussion}

\section{Complexity-determined system pausing vs continuous presentation without pausing (Hypothesis 1)}

The results partially supported the first hypothesis that the complexity-determined system pausing approaches were superior to the continuous presentation without pausing in terms of several measures. The system pausing proposed in this study is determined by the complexity of multimedia presentation sections. In cognitive load theory, the complexity of learning materials is described in terms of element interactivity - a main source of cognitive load. Cognitive overload situations often occur in multimedia presentations with high levels of element interactivity, inhibiting learning outcomes (Sweller, 2010, 2020). Accordingly, pausing the parts of learning content with high element interactivity to give learners more time to deal with the information is undoubtedly the most direct way to reduce potential cognitive overload in multimedia learning.

In the current study, system pauses were positioned either before or after the sections (slides) with high levels of element interactivity based on the theoretical framework of isolated-interacting elements effect (Chen et al., 2017; Lin et al., 2015; Pollock et al., 2002; Sweller, 2010) and segmentation effect (Spanjers et al., 2010; Spanjers et al., 2012). Previous studies of the isolated-interacting elements effect have focused mainly on isolated concepts rather than isolated representations (e.g., Lin et al., 2015). The current study demonstrated the effectiveness of presenting isolated pictorial representations individually in the first place and subsequently presenting the fully interacting representation (pictorial and verbal) after a pause, which extended the application of the isolated-interacting elements effect to the design of paused multimedia environments. 
Providing learners with sufficient time to process, maintain and consolidate the information they have just received is important for successful learning (Spanjers et al., 2010; Spanjers et al., 2012). Inserting pauses after sections with high element interactivity could give participants sufficient time to search the parts of the paused screen mentioned in the previous narration, and then match and integrate the corresponding elements of the pictorial and verbal representations. These activities are important for maintaining and consolidating the studied information before receiving and processing new incoming information.

Element interactivity is a key factor affecting the level of cognitive load of learning materials (Sweller, 2010, 2020); however, the level of element interactivity has seldom been considered in designing pausing approach in multimedia learning. The findings related to the first hypothesis hold significant implication for both researchers and instructional designers. For researchers, the effect of pausing at learning contents with high element interactivity indicates a new and not sufficiently investigated way to benefit learning with dynamic multimedia presentations. The effects of this type of pausing approach need to be verified in more studies. In addition, we recommend further examining if the pauses inserted based on the level of element interactivity have different effects on learners with different levels of prior knowledge. For instructional designers, we recommend evaluating the levels of element interactivity of different sections of learning contents as the first step when designing multimedia presentations. Inserting pauses at sections with high element interactivity could be an efficient and direct way of avoiding potential cognitive overload caused by dynamic multimedia presentations. Learners would be provided with more time to integrate the visual information shown on the screen and the verbal information from the narration, leading to improved learning results.

\section{Learner pausing vs continuous presentation without pausing (Hypothesis 2)}

The results showed that the presentation with learner pausing was more effective than the continuous presentation without pausing, which supported the second hypothesis and echoed the findings of the previous studies (e.g., Hasler et al., 2007; Schwan \& Riempp, 2004). It should be noted that only 10 participants in the learner pausing group pressed the "Pause" button. For the rest of the participants, the most frequently mentioned reasons for not using the "Pause" button were "I don't need to press the button", "I don't have that habit [pressing the pause button]" and "I don't want to press the button". Similar observations were also reported in the studies of Hasler et al. (2007) and Biard et al. (2018), where only a few participants pressed the "Pause" button. For example, in Hasler et al.'s study, a learner pausing group demonstrated better learning performance than a continuous presentation group, even though only one out of 18 participants pressed the "Stop" and "Play" buttons in the required way during learning. The findings related to the second hypothesis again pose this interesting research issue that requires further investigation: why learners demonstrated better learning outcomes when they were allowed to pause the video even if they did not actually pause it.

\section{Complexity-determined system pausing vs learner pausing (Hypothesis 3)}

This study found no significant differences between the complexity-determined system pausing and learner pausing groups in all measures, thus not supporting the third hypothesis. The results were similar to some previous studies comparing the system pausing (by segmentation) and learner pausing instructional conditions (e.g., Hasler et al., 2007). A possible reason is that learner pausing and system pausing had advantages but also shortcomings when applied to multimedia learning. For learner pausing, learners may be in the best position to initiate effective pauses according to their needs, but there is a concern that large individual differences may also affect the learning outcomes (Tabbers \& de Koeijer, 2010). For example, in the current study, the participant in the learner pausing group who had the highest number of pauses did not actually show better learning performance than other participants in the same group. He paused mostly at the sections with low element interactivity, thus demonstrating a limitation of the learner pausing approach.

On the other hand, the system pausing has its strengths. Educators and instructional designers are familiar with the characteristics of learning materials, and the system pauses determined by them are more likely to occur at the most appropriate positions (Castro-Alonso \& Jansen, 2019; Spanjers et al., 2010). However, the system pausing might also induce extraneous cognitive load for some learners who do not need to pause at every position. The findings related to the third hypothesis showed that it is important to consider both the advantages and shortcomings of the system pausing and learner pausing approaches when designing 
dynamic multimedia presentations. In addition, combining the advantages of complexity-determined system pausing with learner pausing might be another potential pausing approach in designing dynamic multimedia presentations. We recommend that future studies evaluate the effectiveness of combining these two pausing types and investigate the potential effects of learners' prior knowledge levels on the effectiveness of pausing.

\section{Conclusions and limitations}

In the current study, two new types of system pausing - the complexity-determined pausing approaches in the design of multimedia presentations were proposed, and their learning effects were evaluated. The results showed that the complexity-determined system pausing is potentially as effective as the learner pausing in terms of overcoming the transient nature of dynamic multimedia presentations and benefiting learning. The system pausing by segmentation has been well recognised and applied to multimedia presentations with clearly structured content (Biard et al., 2018; Mayer \& Chandler, 2001). The current study broadened the scope of this approach by showing the potential of the complexity-determined system pausing in enhancing learning with multimedia presentations. With its focus on sections with high levels of element interactivity rather than just structural units of the learning materials, this approach may contribute to the development of instructional principles for designing cognitively optimal multimedia learning environments. Future studies could investigate the applications of the system pausing determined by element interactivity to other forms of dynamic representations in multimedia learning. The eventual goal would be to develop automated systems (Villagrá-Arnedo et al., 2017) for inserting complexitydetermined system pauses in the learning content based on online, real-time evaluation of its level of element interactivity.

There are serval limitations of this study. First, there is not enough information regarding the participants' views on the different designs of the video. For example, only participants in the learner pausing group who had not paused the video were asked why they had not pressed the "Pause" button during learning. Future studies could conduct a more comprehensive interview following the test phase to obtain more information about participants' perceptions of the different experimental conditions. The second limitation is that since the participants in this study were undergraduates and the topic of mindfulness was rather narrow, the findings of the reported experiment may not generalise to other populations and other learning topics. We recommend that future studies examine the effects of the complexity-determined system pausing approaches with different participants using different learning topics.

\section{Acknowledgements}

We would like to thank the editor of the Australasian Journal of Educational Technology and anonymous reviewers, who provided all the valuable comments and suggestions. Also, we would like to express our gratitude to the Ministry of Science and Technology (MOST) in Taiwan for its financial support to this research under Grant No. MOST 106-2511-S-003-033-MY3 and MOST 108-2511-H-003-044MY4. Moreover, we sincerely appreciate the funding offered by the Institute for Research Excellence in Learning Sciences of the National Taiwan Normal University from the Featured Areas Research Center Program within the framework of the Higher Education Sprout Project by the Ministry of Education in Taiwan. Finally, we would like to thank all the people who helped us and supported this research. This research would not have been possible without them.

\section{References}

Ayres, P., \& Paas, F. (2007). Making instructional animations more effective: A cognitive load approach. Applied Cognitive Psychology, 21(6), 695-700. https://doi.org/10.1002/acp.1343

Barrouillet, P., \& Camos, V. (2007). The time-based resource sharing model of working memory. In N. Osaka, R. H. Logie, \& M. D'Esposito (Eds.), The cognitive neuroscience of working memory (pp. 5980). Oxford University Press. https://doi.org/10.1093/acprof:oso/9780198570394.003.0004

Berney, S., \& Bétrancourt, M. (2016). Does animation enhance learning? A meta-analysis. Computers \& Education, 101, 150-167. https://doi.org/10.1016/j.compedu.2016.06.005 
Bétrancourt, M. (2005). The animation and interactivity principles in multimedia learning. In R. E. Mayer (Ed.), The Cambridge handbook of multimedia learning (pp. 111-146). Cambridge University Press. https://doi.org/10.1017/ CBO9780511816819.004

Biard, N., Cojean, S., \& Jamet, E. (2018). Effects of segmentation and pacing on procedural learning by video. Computers in Human Behavior, 89, 411-417. https://doi.org/10.1016/j.chb.2017.12.002

Castro-Alonso, J. C., de Koning, B. B., Fiorella, L., \& Paas, F. (2021). Five strategies for optimizing instructional materials: Instructor-and learner-managed cognitive load. Educational Psychology Review. https://doi.org/10.1007/s10648-021-09606-9

Castro-Alonso, J. C., \& Jansen, P. (2019). Sex differences in visuospatial processing. In J. C. CastroAlonso (Ed.), Visuospatial processing for education in health and natural sciences (pp. 81-110). Springer. https://doi.org/10.1007/978-3-030-20969-8.

Chen, O., Castro-Alonso, J. C., Paas, F., \& Sweller, J. (2018). Extending cognitive load theory to incorporate working memory resource depletion: Evidence from the spacing effect. Educational Psychology Review, 30, 483-501. https://doi.org/10.1007/s10648-017-9426-2

Chen, O., Woolcott, G., \& Sweller, J. (2017). Using cognitive load theory to structure computer-based learning including MOOCs. Journal of Computer Assisted Learning, 33(4), 293-305. https://doi.org/10.1111/jcal.12188

Fraenkel, J. R.,Wallen, N. E., \& Hyun, H. (2011). How to design and evaluate research in education (8th ed.). McGraw Hill.

Furr, R. M., \& Rosenthal, R. (2003). Evaluating theories efficiently: The nuts and bolts of contrast analysis. Understanding Statistics, 2(1), 33-67. https://doi.org/10.1207/S15328031US0201_03

Hasler, B. S., Kersten, B., \& Sweller, J. (2007). Learner control, cognitive load and instructional animation. Applied Cognitive Psychology, 21(6), 713-729. https://doi.org/10.1002/acp.1345

Höffler, T. N., \& Schwartz, R. N. (2011). Effects of pacing and cognitive style across dynamic and nondynamic representations. Computers \& Education, 57(2), 1716-1726. https://doi.org/10.1016/j.compedu.2011.03.012

Kalyuga, S. (2009). Managing cognitive load in adaptive multimedia learning. IGI Global. https://doi.org/10.4018/978-1-60566-048-6

Khacharem, A., Zoudji, B., \& Kalyuga, S. (2015). Expertise reversal for different forms of instructional designs in dynamic visual representations. British Journal of Educational Technology, 46(4), 756767. https://doi.org/10.1111/bjet.12167

Leahy, W., \& Sweller, J. (2011). Cognitive load theory, modality of presentation and the transient information effect. Applied Cognitive Psychology, 25(6), 943-951. https://doi.org/10.1002/acp.1787

Lee, J. Y., Donkers, J., Jarodzka, H., Sellenraad, G., \& van Merriënboer, J. J. (2020). Different effects of pausing on cognitive load in a medical simulation game. Computers in Human Behavior, 110, Article 106385. https://doi.org/10.1016/j.chb.2020.106385

Lin, Y. C., Liu, T. C., \& Sweller, J. (2015). Improving the frame design of computer simulations for learning: Determining the primacy of the isolated elements or the transient information effects. Computers \& Education, 88, 280-291. https://doi.org/10.1016/j.compedu.2015.06.001

Liu, T. C., Lin, Y. C., \& Paas, F. (2021). A new application of the temporal contiguity effect in designing narrated PowerPoint presentation [Manuscript submitted for publication].

Mayer, R. E., \& Chandler, P. (2001). When learning is just a click away: Does simple user interaction foster deeper understanding of multimedia messages? Journal of Educational Psychology, 93(2), 390397. https://doi.org/10.1037/0022-0663.93.2.390

Merkt, M., Ballmann, A., Felfeli, J., \& Schwan, S. (2018). Pauses in educational videos: Testing the transience explanation against the structuring explanation. Computers in Human Behavior, 89, 399410. https://doi.org/10.1016/j.chb.2018.01.013

Paas, F., \& Van Merriënboer, J. J. G. (1993). The efficiency of instructional conditions: an approach to combine mental-effort and performance measures. Human Factors, 35(4), 737-743. https://doi.org/10.1177/001872089303500412

Paas, F., \& Van Merriënboer, J. J. G. (1994). Variability of worked examples and transfer of geometrical problem-solving skills: a cognitive-load approach. Journal of Educational Psychology, 86(1), 122133. https://doi.org/10.1037/0022-0663.86.1.122

Pollock, E., Chandler, P., \& Sweller, J. (2002). Assimilating complex information. Learning and Instruction, 12(1), 61-86. https://doi.org/10.1016/S0959-4752(01)00016-0

Rosenthal, R., \& Rosnow, R. L. (1985). Contrast analysis: Focused comparisons in the analysis of variance. Cambridge University Press. https://doi.org/10.2307/2289419 
Schwan, S., \& Riempp, R. (2004). The cognitive benefits of interactive videos: learning to tie nautical knots. Learning and Instruction, 14(3), 293-305. https://doi.org/10.1016/j.learninstruc.2004.06.005

Seltman, H. J. (2018). Experimental design and analysis. http://www.stat.cmu.edu/ hseltman/309/Book/Book.pdf

Spanjers, I. A., Van Gog, T., \& van Merriënboer, J. J. (2010). A theoretical analysis of how segmentation of dynamic visualizations optimizes students' learning. Educational Psychology Review, 22(4), 411423. https://doi.org/10.1007/s10648-010-9135-6

Spanjers, I. A., van Gog, T., Wouters, P., \& van Merriënboer, J. J. (2012). Explaining the segmentation effect in learning from animations: The role of pausing and temporal cueing. Computers \& Education, 59(2), 274-280. https://doi.org/10.1007/s10648-010-9135-6

Sweller, J. (2010). Element interactivity and intrinsic, extraneous, and germane cognitive load. Educational Psychology Review, 22, 123-138. https://doi.org/10.1007/s10648-010-9128-5

Sweller, J. (2020). Cognitive load theory and educational technology. Educational Technology Research and Development, 68, 1-16. https://doi.org/10.1007/s11423-019-09701-3

Sweller, J., Ayres, P., \& Kalyuga, S. (2011). Explorations in the learning sciences, instructional systems and performance technologies: Vol. 1. Cognitive load theory. Springer. https://doi.org/10.1007/978-14419-8126-4

Tabbers, H. K., \& de Koeijer, B. (2010). Learner control in animated multimedia instructions. Instructional Science, 38, 441-453. https://doi.org/10.1007/s11251-009-9119-4

Van Merriënboer, J. J. G., \& Kester, L. (2014). The four-component instructional design model: Multimedia principles in environments for complex learning. In R. E. Mayer (Ed.), The Cambridge handbook of multimedia learning (pp. 104-148). Cambridge University Press. https://doi.org/10.1017/CBO9781139547369.007

Villagrá-Arnedo, C. J., Gallego-Durán, F. J., Llorens-Largo, F., Compañ-Rosique, P., Satorre-Cuerda, R., \& Molina-Carmona, R. (2017). Improving the expressiveness of black-box models for predicting student performance. Computers in Human Behavior, 72, 621-631. https://doi.org/10.1016/j.chb.2016.09.001

Wong, M., Castro-Alonso, J. C., Ayres, P., \& Pass, F. (2020). The effects of transient information and element interactivity on learning from instructional animations. In S. Tindall-Ford, S. Agostinho, \& J. Sweller (Eds.), Advances in cognitive load theory (pp. 80-88). Routledge. https://doi.org/10.4324/9780429283895-7

Wouters, P., Tabbers, H. K., \& Paas, F. (2007). Interactivity in video-based models. Educational Psychology Review, 19, 327-342. https://doi.org/10.1007/s10648-007-9045-4

Corresponding author: Tzu-Chien Liu, tzuchien@ @ ntnu.edu.tw

Copyright: Articles published in the Australasian Journal of Educational Technology (AJET) are available under Creative Commons Attribution Non-Commercial No Derivatives Licence (CC BY-NC-ND 4.0). Authors retain copyright in their work and grant AJET right of first publication under CC BY-NC-ND 4.0 .

Please cite as: Liu, T.-C., Lin, Y.-C., \& Kalyuga, S. (2022). Effects of complexity-determined system pausing on learning from multimedia presentations. Australasian Journal of Educational Technology, 38(1), 102-114. https://doi.org/10.14742/ajet.7267 